\title{
Design and Application of Detection Method for Soft and Hard Points of Intelligent Cigarette Maker
}

\author{
Yuliang Li ${ }^{1, a}$, Shuai Yang ${ }^{2, b}$, Lihong Zhang ${ }^{1, \mathrm{c}}$ and Qiang Zhu ${ }^{2, \mathrm{~d}}$ \\ ${ }^{1}$ Hangzhou Cigarette Factory, ZJTI, Kehai Road 118, Hangzhou, China. \\ 2 Technical Center, ZJTI, Kehai Road 118, Hangzhou, China. \\ aliyuliang@zjtobacco.com, byangshuai@zjtobacco.com, \\ czhanglh@zjtobacco.com, dzhuqiang@zjtobacco.com
}

\section{Keywords: Cigarette Maker; Soft Point; Hard Point; Detection}

\begin{abstract}
Aiming at the problem that the existing cigarette machine detection technology has low precision in detecting the soft and hard points of cigarettes in the cigarette production process, an intelligent online soft and hard point detection method of the cigarette machine is designed. The method has the following characteristics: 1 . Segmented detection; 2. the number of segments is adjustable, the length of the segment is adjustable; 3 . the threshold can be adjusted in real time; 4 . adjustable baseline. The application results show that this method has the advantages of accurate calculation, rapid response, and convenient adjustment. The detection accuracy rate is more than 95\%, which has played a positive role in reducing the consumption of cigarette machines.
\end{abstract}

\section{Introduction}

The uniformity of the density distribution of cigarettes in tobacco rods the stability of cigarette physical indicators (suction resistance, hardness, etc.), smoke chemical indicators (tar, nicotine, carbon monoxide, etc.) and sensory quality (openness, clustering, etc.) Important factors. In the cigarette manufacturing process, due to factors such as the uniformity of the tobacco (such as wrapping, wet mass, and stalk) and other factors, negative pressure fluctuations in the plenum and other equipment factors, the density of tobacco in a certain section of the cigarette may be too large or too small. The phenomenon of hard point, soft point (1).

The current device detection methods for hard and soft points are: The overall average density of each cigarette, and the absolute limit percentage of hard points and soft points under artificially set standard density, and the pair is beyond the limit during the production process. Products that exceed the rejection limit should be rejected (2).

However, in the actual production process, this detection method has a high false detection rate, a high dropout rate, and poor practicality, which in turn causes abnormal consumption.

The uniformity of the density distribution of cigarettes in tobacco rods the stability of cigarette physical indicators (suction resistance, hardness, etc.), smoke chemical indicators (tar, nicotine, carbon monoxide, etc.) and sensory quality (openness, clustering, etc.) Important factors. In the cigarette manufacturing process, due to factors such as the uniformity of the tobacco (such as wrapping, wet mass, and stalk) and other factors, negative pressure fluctuations in the plenum and other equipment factors, the density of tobacco in a certain section of the cigarette may be too large or too small. The phenomenon of hard point, soft point (1).

The current device detection methods for hard and soft points are: The overall average density of each cigarette, and the absolute limit percentage of hard points and soft points under artificially set standard density, and the pair is beyond the limit during the production process. Products that exceed the rejection limit should be rejected (2). 
However, in the actual production process, this detection method has a high false detection rate, a high dropout rate, and poor practicality, which in turn causes abnormal consumption.

The Formation of Cigarette soft and Hard Points. The uniformity of the density distribution of cigarettes in tobacco rods the stability of cigarette physical indicators (suction resistance, hardness, etc.), smoke chemical indicators (tar, nicotine, carbon monoxide, etc.) and sensory quality (openness, clustering, etc.) Important factors. In the cigarette manufacturing process, due to factors such as the uniformity of the tobacco (such as wrapping, wet mass, and stalk) and other factors, negative pressure fluctuations in the plenum and other equipment factors, the density of tobacco in a certain section of the cigarette may be too large or too small. The phenomenon of hard point, soft point (1).

The current device detection methods for hard and soft points are: The overall average density of each cigarette, and the absolute limit percentage of hard points and soft points under artificially set standard density, and the pair is beyond the limit during the production process. Products that exceed the rejection limit should be rejected (2). However, in the actual production process, this detection method has a high false detection rate, a high dropout rate, and poor practicality, which in turn causes abnormal consumption.

The Lack of Soft and Hard Point Existing Detection Method. In the former industry, the general detection methods for cigarettes and soft cigarettes in cigarette equipment are: the overall average density of each cigarette, and the absolute limit percentage of hard dots and soft dots under the artificially set standard density, which exceeds the production process. The elimination of soft and hard cigarettes. However, in the actual production process, this test method has a high false-rejection rate, low dropout rate, and poor practicality. It is mainly reflected in the following two points (4):

The first is the inaccuracy of the test reference, and the different sizes of the cigarette end-counter increments are different for the different dresser specifications (flattener groove length and depth), and the soft and hard points are all based on the density standard percentage baseline. In the test, the tobacco shreds in the cigarettes themselves have a bowl-shaped distribution with higher density at both ends and a smaller density in the middle, and the equipment only judges whether the cigarette is a cigarette with hard point or with soft point based on the absolute percentage of the theoretical average density, causing a large number of cigarettes to be missed or missed. For example, the same size of wet tobacco shreds can be easily removed if they are distributed at both ends of the cigarette. If it is distributed in the middle section of the cigarette, it is difficult to remove.

The second is the irrationality of the limit adjustment. When the mechanical structure of the equipment such as the specifications of the leveler is determined, the increase in the density of the cigarette tip is determined by the process parameters such as the raw material (filling value of tobacco, etc.) and the equipment (negative pressure in the chamber, etc.) during the production process. In the process, there are fluctuations in such process parameters, and only relying on human-made adjustments cannot satisfy the accuracy of the real-time adjustment of the parameters. This can also cause a large number of cigarettes to be missed or missed.

\section{Method Design}

Working Flow. According to the all above, in order to achieve accurate detection of soft and hard points during cigarette production, microwave density signals must be collected and then signal processing performed. The technical approach we adopt is as follows: 


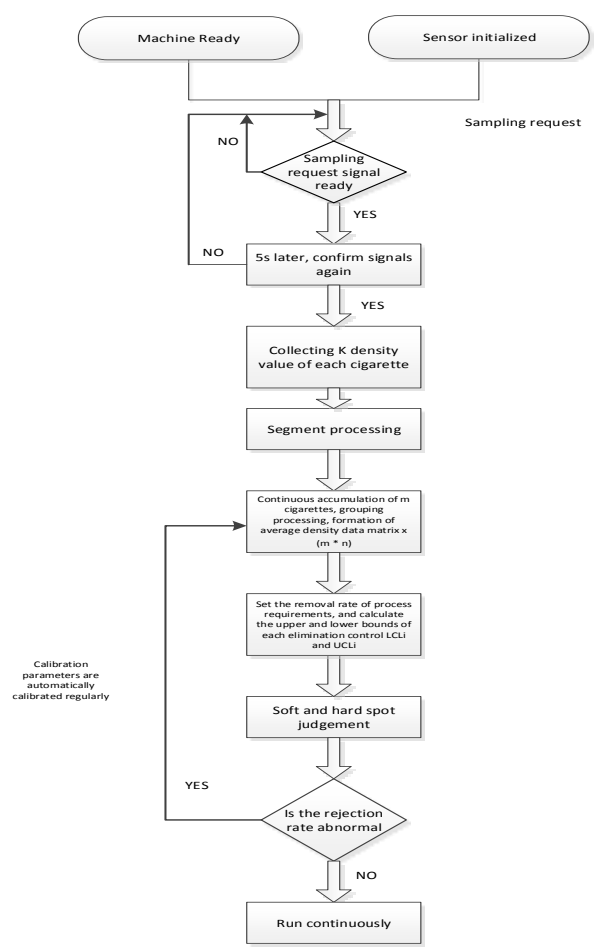

Figure 1. Finite Working Flow

\section{Method Principle.}

1. During the cigarette production process, the high-speed sampling of the density value of each cigarette is performed periodically (5). The sampling data is obtained. Each cigarette can obtain $\mathrm{k}$ density sampling data $\mathrm{d} 1 \sim \mathrm{dk}$, and the arithmetic average of $\mathrm{d} 1 \sim \mathrm{dk}$ is calculated. The average value is the density value of the whole cigarette.

2. The sampling data of each cigarette $\mathrm{k}$ is divided into 1 ...n segments processing (where $\mathrm{n}$ is selected: $\mathrm{k}$ can be divisible by $\mathrm{n}$ ), and the average density of each segment is calculated using an arithmetic average to obtain a segmentation. The average density array $\operatorname{Di}(\mathrm{i}=1, \ldots, \mathrm{n})$ for each post-segment.

3. The group average density array $\mathrm{Di}$ is grouped and cumulatively preprocessed, and the cumulative number of cigarettes in each group is set to be $\mathrm{m}$ branches, and the average density data matrix of $m$ cigarettes in the segmented mode is obtained as $X(m \times n)$, and always carry out moving average, that is, the average object of each segment of the $n$ segments of each cigarette is the average object of each segment of the $n$ segments of the former $m$ cigarettes.

4. According to the process requirements and the basic principles of quality management, the total elimination percentage requirement for soft and hard points is $y \%$. Through the actual production of batch cigarettes, the average density of each segment and the upper and lower limit settings LCLi $(\mathrm{i}=1, \ldots, \mathrm{n}) \%$ and $\mathrm{UCLi}(\mathrm{i}=1, \ldots, \mathrm{n}) \%$, that is, $\mathrm{UCL}$ above the control upper limit represents that there is a hard point anomaly in the segment, and below the lower limit represents that there is a soft point anomaly in the segment.

5. When on-line application, real-time acquisition of $\mathrm{k}$ density sampling data for each cigarette, and calculating the average density of segments in $\mathrm{n}$ segments, comparing the segment average density of each segment with the control upper and lower limits of the segment, if only there is $\mathrm{j}$ segment ( The data exceeding $1 \leq \mathrm{j} \leq \mathrm{n}$ ) now indicates that the cigarettes in this section are qualified smokes with soft points and hard point anomalies.

6. The density matrix and the upper and lower limit calculation method, wherein the density matrix $\mathrm{X}$ may be re-extracted at a fixed time $\mathrm{T}$, and the average density of the segments and the upper and lower limits may be re-calculated to implement regular automatic parameter checking. 


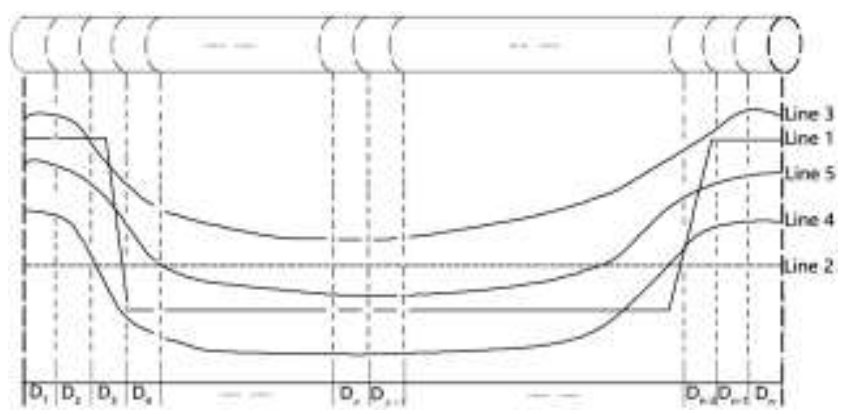

Figure 2. Finite Cigarette Density Distrubution

Application Effection Method Principle. In order to verify the accuracy of the method, the cigarette soft and hard point sampling and picking up the removed cigarette branch can be used to verify the effect. The instrument used is the TEWS MW4420 cigarette smoke density detector with a sample size of 100 and 10 groups. The result is as follows:

Table 1 Accuracy test results

\begin{tabular}{ccc}
\hline $\begin{array}{c}\text { Sample } \\
\text { Group }\end{array}$ & $\begin{array}{c}\text { Accuracy of } \\
\text { Traditional } \\
\text { Detection Method }\end{array}$ & $\begin{array}{c}\text { Accuracy of } \\
\text { Intelligent } \\
\text { Detection Method }\end{array}$ \\
\hline 1 & $52 \%$ & $95 \%$ \\
\hline 2 & $61 \%$ & $97 \%$ \\
\hline 3 & $45 \%$ & $100 \%$ \\
\hline 4 & $41 \%$ & $98 \%$ \\
\hline 5 & $63 \%$ & $95 \%$ \\
\hline 6 & $70 \%$ & $96 \%$ \\
\hline 7 & $52 \%$ & $99 \%$ \\
\hline 8 & $65 \%$ & $97 \%$ \\
\hline 9 & $43 \%$ & $98 \%$ \\
\hline 10 & $57 \%$ & $100 \%$ \\
\hline Average accuracy & $55 \%$ & $98 \%$ \\
\hline $\begin{array}{c}\text { Accuracy standard } \\
\text { deviation }\end{array}$ & $9.4 \%$ & $1.7 \%$ \\
\hline
\end{tabular}

\section{Conclusion}

Based on the above method of cigarette online soft and hard point detection method, first obtain the density information of $\mathrm{n}$ points of each cigarette, divide it into segments, calculate the moving average, and then divide the density values of $\mathrm{n}$ points of the current cigarette branch. The moving average of the $\mathrm{n}$ points of the cigarette before the segment method is compared to detect the soft and hard points and guide the control system to reject. The signal processing of this method is accurate, and the coincidence rate with the real object is more than $98 \%$.

\section{Acknowledgements}

The authors would like to acknowledge the support of funding from Hangzhou Cigarette Factory, Zhejiang Tobacco of Industry, China. 


\section{References}

[1] ZHANG Jimin, QIN Hua. Measurement of moisture and density distribution of cigarettes(J). Industrial Instrumentation and Automation Devices, 2014(04):90-91+95.

[2] DAI Yongsheng, YAO Wenxiang, WU Kai, BAO Zhihua, PENG Liming. Effect of Equipment Parameters on the Distribution of Cut Tobacco in Tobacco Branches during Rolling Process $(\mathrm{J})$. Tobacco Science, 2012(04):9-12.

[3] HUANG Wei. Application of Microwave Detection Technology in Tobacco Weight Control System(J). Tobacco Science, 2006(03):15-18.

[4] XIONG Anyan, LI Chunguang. Effect of different specification levelers on the quality of cigarettes in ZJ17 maker(J). Tobacco Science, 2011(11):14-18.

[5] LI Jinxue, GAO Zunhua, YANG Fan. Influence of Distribution of Cut Tobacco on Cigarette Quality in Cigarette Branch. Tobacco Science,2004(08):11-12+15. J. van der Geer, J.A.J. Hanraads, R.A. Lupton, The art of writing a scientific article, J. Sci. Commun. 163 (2000) 51-59. 